\title{
Elevator illusion: Influences of otolith organ activity and neck proprioception*
}

\author{
MALCOLM M. COHEN \\ Naval Air Development Center, Warminster, Pennsylvania 18974
}

\begin{abstract}
Each of nine Ss experienced gravitational-inertial forces (GIFs) of $1.00,1.25,1.50$, and $1.75 \mathrm{G}_{\mathrm{z}}$ with his head erect and pitched forward at angles of 15 and $30 \mathrm{deg}$. The magnitude of the elevator illusion was shown to depend on both the intensity of the GIFs and the orientation of the head. Contributions of otolith organ activity and of neck proprioception to the ilusion were examined, and theoretical bases for their influences were discussed.
\end{abstract}

Stationary visible objects appear to rise when gravitational-inertial forces (GIFs) are increased in magnitude along an $O$ 's head-to-foot, or $G_{z}$, body axis (von Beckh, 1956; Clark, Graybiel, \& MacCorquodale, 1948; M. Cohen, 1970a, b; Graybiel, Clark, \& MacCorquodale, 1947; Niven, Whiteside, \& Graybiel, 1963; Roman, Warren, Niven, \& Graybiel, 1963; Whiteside, 1961). The change of apparent elevation that results when GIFs are altered in magnitude alone has been termed the "elevator illusion" (Whiteside, 1961); the elevator illusion is similar to the oculogravic illusion (Graybiel, 1952), an illusion that results when GIFs are altered in both magnitude and direction (Niven et al, 1963). The distinction between the elevator illusion and the oculogravic illusion has been based on differences in the conditions under which they are generated, and although both illusions have been studied extensively in recent years (Brandt \& Fluur, 1967a, b; Correia, Hixson, \& Niven, 1968; Graybiel, 1952; Niven et al, 1963; Roman et al, 1963; Schone, 1964), no comprehensive explanation of their underlying mechanisms has been developed.

Oculomotor reflexes, resulting from stimulation of the otolith organs by GIFs are important in both illusions. For example, Fluur (1970) has demonstrated that direct electrical stimulation of the otolith organs will cause the position of the eyes to deviate. However, when caused by altered GIFs, the angular deviation in the position of the eyes is significantly smaller than the angular deviation in the apparent elevation of a visual target (Brandt \& Fluur, 1967a,b). Thus, otolith-oculomotor reflexes alone are insufficient to account for the illusions.

Although labyrinthine receptors play a contributory role in the oculogravic illusion, tactile, muscular, and proprioceptive receptors are also involved (Clark \& Graybiel, 1968). When Os with damaged labyrinthine receptors experience GIFs that are altered in both magnitude and direction, they report the oculogravic illusion. in a modified form (Graybiel \& Clark, 1965),

*Supported by funds under Independent Research Project ZR0000101, Work Unit CS-6-01, at the Naval Air Development Center. but when they experience GIFs that are altered in magnitude alone, they do not report the elevator illusion at all (Niven et al, 1963). These findings suggest that labyrinthine activity is important for the oculogravic illusion, but necessary for the elevator illusion.

Anatomical studies have shown that the macular surface of the utricle, a surface in the labyrinth consisting largely of hair-cell receptors, slopes anteriorly upward by approximately $30 \mathrm{deg}$ from the frontal-occipital axis of the head (Corvera, Hallpike, \& Schuster, 1958). If stimulation of the utricle is accomplished by shearing forces along the macular surface (Schone, 1964), or by a combination of shearing forces and compression (Correia et al, 1968), then changes in the orientation of the head relative to GIFs should produce changes in stimulation of the utricle. Further, if the apparent elevation of visual objects depends on stimulation of the utricle, then the apparent elevation of visual objects should be different for different orientations of the head.

To minimize changes in the apparent elevation of visual objects when the orientation of the head is changed, signals from proprioceptors in the neck serve to adjust the position of the eyes (L. Cohen, 1961; Cope \& Ryan, 1959; de Kleyn, 1918). For example, de Kleyn (1918) has shown that reflex changes in eye position remain after bilateral extirpation of labyrinthine receptors; when extirpation is followed by cervical lesions, the reflexes are eliminated. Similarly, Cope and Ryan (1959) have noted that cervical diseases, which leave the labyrinthine receptors unaffected, often result in vertigo and disorientation. Thus, in the normal terrestrial environment, it appears that the signals from proprioceptors in the neck are balanced with signals from the otolith organs to allow the apparent (and retinal) position of visual objects to remain relatively stable for different orientations of the head. When GIFs are altered, the delicate balance is disrupted, and visual objects appear to move whenever the orientation of the head is changed (Guedry, 1968). However, the separate and independent influences of neck proprioception and otolith organ activity in the perceived elevation of visual objects remain to be quantified. 


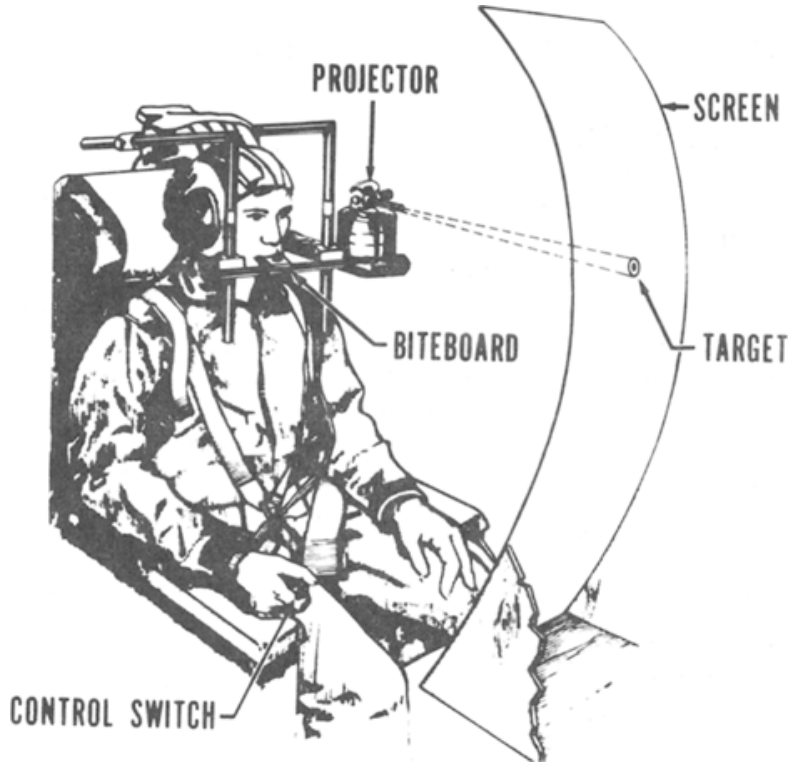

Fig. 1. Apparatus used to measure the apparent elevation of a visual target.

The present study attempts to separate the effects of neck proprioception and otolith organ activity in the elevator illusion. It is assumed that neck proprioception is altered when the head is pitched with respect to the trunk. It is also assumed that the activity of the otolith organs is altered either when the orientation of the otolith organs is changed with respect to the GIFs or, for most orientations of the head, when the magnitude of the GIFs is changed. This study examines the illusion produced by four magnitudes of GIFs with the head oriented in each of three positions.

\section{METHOD}

\section{Subjects}

Nine male L.S. Naval personnel, between the ages of 19 and 32 years, served as volunteer $S s$. All had previous experience in riding the human centrifuge and performing control tasks similar to the one used in this study. They had normal visual acuity, and none showed any evidence of otic deficiency.

\section{Experimental Conditions}

The conditions of the experiment were defined by the GIFs in the $G_{2}$ direction, along the head-to-foot axis of $S$ 's body parallel to his spine, and by the orientation of S's head.

The Naval Air Development Center's human centrifuge (Christy, 1949; Crosbie, 1956, 1967) was used to generate the GIFs. Each $S$ was seated in the gondola of the centrifuge and was exposed to GIFs of $1.00,1.25,1.50$, and $1.75 \mathrm{G}_{\mathrm{z}}$. These values, expressed as multiples of the earth's gravitational force, represent the vectorial sum of gravity and the radial force generated by rotation of the centrifuge arm.

The GIFs were determined by calculation from the angular velocity $(\omega)$ of the centrifuge arm and the distance $(R)$ of S's head from the center of rotation, according to the equation:

$$
\operatorname{GIF}\left(G_{z} \text { units }\right)=\frac{\sqrt{\left(\omega^{2} R\right)^{2}+(9.81)^{2}}}{9.81}
$$

where $\omega$ is in radians/second. $\mathrm{R}$ is in meters, and the constant. 9.81 , is the acceleration ciue to the earth's gravity in meters/second ${ }^{2}$. Accelerometers mounted at the center of the gondola indicated GIFs within $1 \%$ of those calculated by Eq. 1 .

Each $S$ used a custom-fitted dental impression biteboard to position his head in each of three orientations: erect $(0 \mathrm{deg})$, pitched $15 \mathrm{deg}$ forward, and pitched $30 \mathrm{deg}$ forward. The orientations were defined by the angle between a horizontal plane and Reid's baseline, the anterior-posterior axis of the head that may be visualized as a straight line connecting the center of the ear canal with the bottom of the orbit of the eyes (Corvera et al, 1958).

The four different GIFs were combined with the three orientations of the head to provide 12 different experimental conditions. The 12 conditions, randomized in order, were presented to each $S$ in separate sessions; no $S$ was given more than four sessions on any day.

Each experimental session lasted for $7 \mathrm{~min}$ and consisted of five parts: (1) a preexposure period of $2 \min$ at $1.00 \mathrm{G}_{z}$; (2) a transition period of $30 \mathrm{sec}$ in which $G_{z}$ was increased along a haversine curve from $1.00 \mathrm{G}_{\mathbf{z}}$ to the experimental level; (3) an exposure period of $2 \mathrm{~min}$ at a steady $\mathrm{G}_{2} ;(4)$ a transition period of $30 \mathrm{sec}$ in which $G_{z}$ was decreased along a haversine curve from the experimental level to $1.00 \mathrm{G}_{\mathrm{z}}$; and (5) a postexposure period of $2 \mathrm{~min}$ at $1.00 \mathrm{G}_{\mathrm{z}}$.

\section{Apparatus and Measurements}

At the beginning of each experimental session, $\mathrm{S}$ was strapped firmly in his seat by a shoulder harness and lap belt; his head was appropriately positioned by the biteboard; the lights of the gondola were extinguished, and the hatch was tightly sealed.

The apparatus, illustrated in Fig. 1, provided $S$ with a visual target that was projected on the screen before him in the otherwise totally darkened gondola. The target was an annulus of light with an outer diameter of $25 \mathrm{~mm}$ and a thickness of $5 \mathrm{~mm}$. At the viewing distance of $92 \mathrm{~cm}$, the target subtended approximately $1.6 \mathrm{deg}$ of visual angle. A servomotor continuously drove the projected target either up or down on the screen at a rate of $5 \mathrm{deg} / \mathrm{sec}$, and $S$ operated a control switch that reversed the direction in which the target was moving. By repeatedly activating the control switch, $S$ continuously bracketed the position that he considered to be at the earth's horizon.

Signals from the servomotor, continuously monitored on strip chart and magnetic tape recordings, indicated the position of the target on the screen. The signals, processed on an EAI analog computer (Model 231-R), were electronically averaged over successive $30-\mathrm{sec}$ intervals to provide measures of target elevation during each experimental session.

\section{RESULTS AND DISCUSSION}

Figure 2 illustrates the changes of target elevation that result from exposure under each of the experimental conditions. The data, representing the mean elevation of the target computed across all nine Ss, are typical of individuals as well.

When the head was erect and when it was pitched $15 \mathrm{deg}$ forward, $S$ lowered the target by increasing amounts as the GIFs increased. Since $S$ attempted to keep the target at the horizon, a stationary target would appear to rise under these conditions, and the greater the GIFs, the higher the apparent elevation of the target.

In contrast, when the head was pitched $30 \mathrm{deg}$ forward, $S$ did not lower the target as the GIFs increased. When the head is pitched 30 deg forward, the 
macular surface of the utricle is approximately horizontal and perpendicular to GIFs applied along the $\mathrm{G}_{\mathrm{z}}$ body axis (Corvera et al, 1958). Under these conditions, shearing forces are virtually eliminated at the macular surface of the utricle. If shearing forces are necessary to stimulate the utricle, and if the apparent elevation of the target depends on utricular stimulation (Schone, 1964), the 30-deg forward pitch of the head should allow increased GIFs without changes in apparent elevation.

The relationship between the angle at which the head is pitched and the apparent elevation of the target for given GIFs is summarized in Fig. 3. The data, computed across all nine Ss, indicate the magnitude of the elevator illusion, i.e., where a stationary target at the horizon would appear if Ss did not adjust its position. Each data point represents the mean of the four $30-\mathrm{sec}$ average target elevations recorded during the exposure period.

The analysis of variance summarized in Table 1 clearly indicates that both head pitch and $G_{z}$, as well as their interaction, contribute significantly to the elevator illusion.

Two aspects of the data are particularly important. First, at the levels of $\mathrm{G}_{z}$ examined here, the target appears lower when the head is pitched forward than when it is erect. Schone (1964) also reported that visual objects appear lower when the head is pitched forward, but only when GIFs are greater than $1.00 \mathrm{G}_{\mathbf{z}}$. According to Schone, the apparent position of visible objects remains constant in a $1.00-\mathrm{G}_{\mathrm{z}}$ environment, irrespective of the orientation of the head. According to these data, and those of DeLage (1886), apparent position is not constant for different orientations of the head, even in a $1.00-G_{\mathrm{z}}$ environment. This finding suggests that the influences of neck proprioception and otolith organ

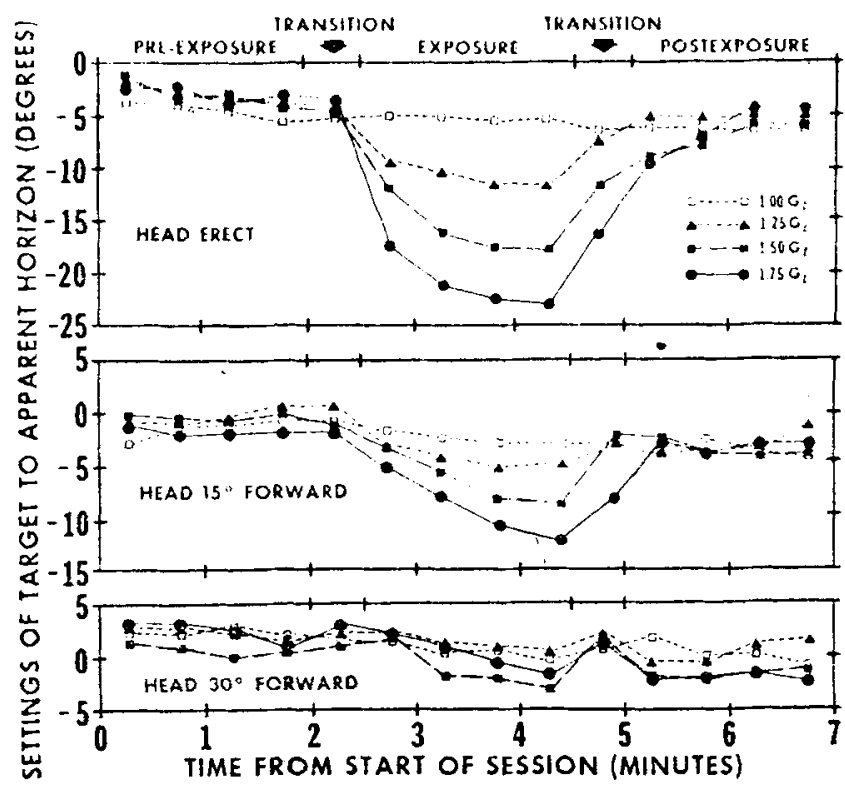

Fig. 2. Settings of the target to the apparent horizon during experimental sessions.

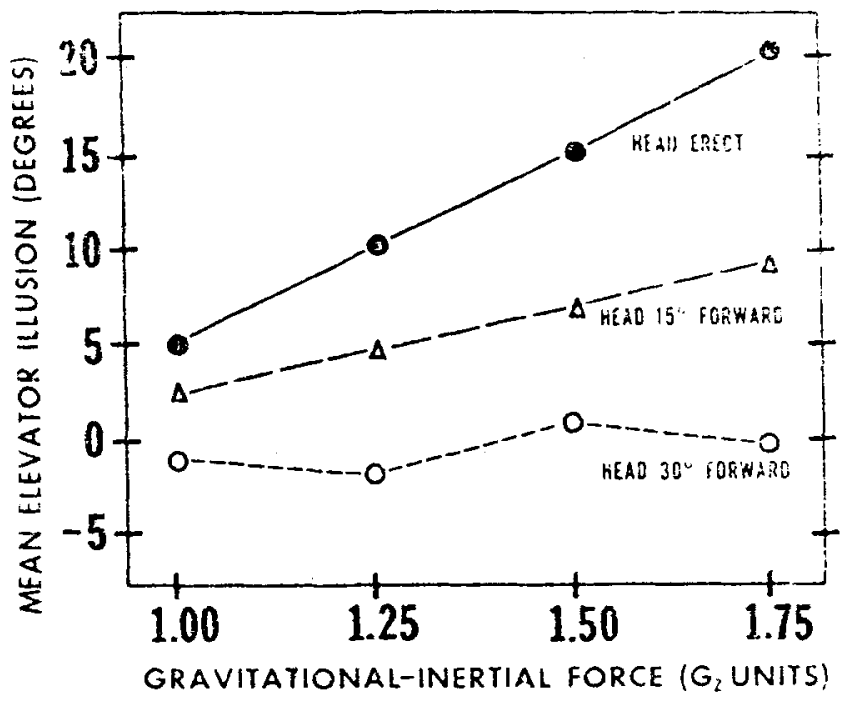

Fig. 3. Mean elevator illusion during exposure to gravitational-inertial forces for three orientations of the head.

Table 1

Analysis of Variance: Elevator Illusion During Exposure to Steady State $G_{Z}$

\begin{tabular}{lrrrrc}
\multicolumn{1}{c}{ Source } & df & \multicolumn{1}{c}{$\begin{array}{c}\text { Sum } \\
\text { Squares }\end{array}$} & $\begin{array}{c}\text { Mean } \\
\text { Square }\end{array}$ & F Ratio & p \\
\hline Head Pitch & 2 & 3251.63 & 1625.82 & $17.00^{*}$ & $<.001$ \\
$\mathrm{G}_{\mathrm{Z}}$ & 3 & 962.56 & 320.85 & $12.35^{* *}$ & $<.001$ \\
Head Pitch by $\mathrm{G}_{\mathrm{Z}}$ & 6 & 490.06 & 81.68 & $3.99 \dagger$ & $<.01$ \\
Subjects (S) & 8 & 2024.97 & 253.12 & -- & -- \\
Head Pitch by S & 16 & 1529.72 & 95.61 & -- & -- \\
$\mathrm{G}_{Z}$ by $\mathrm{S}$ & 24 & 623.73 & 25.99 & -- & -- \\
Head Pitch & 48 & 981.52 & 20.45 & - & -- \\
by $\mathrm{G}_{\mathrm{Z}}$ by S & 4 & & & &
\end{tabular}

* Tested with head pitch by $S$ as error term.

**Tested with $G_{z}$ by $S$ as error term.

tTested with head pitch by $G_{z}$ by $S$ as error term.

activity are not precisely balanced in a $1.00-G_{z}$ environment, and that position constancy, the apparent stability of seen objects when the head moves, is only approximate. Second, the rate at which the elevator illusion changes with increased $G_{z}$ is reduced as the head is pitched forward on the trunk. With the head erect, the elevator illusion changes at a mean rate of $20.4 \mathrm{deg} / \mathrm{G}_{2}$ as determined by a method of least squares linear fit to the data; with the head pitched $15 \mathrm{deg}$ forward, the elevator illusion changes at a mean rate of $9.1 \mathrm{deg} / \mathrm{G}_{\mathrm{z}}$; with the head pitched $30 \mathrm{deg}$ forward, the elevator illusion does not change significantly with increased $G_{z}$ The mean rates of change of the elevator illusion are significantly different for the different orientations of the head, $F(2,16)=9.35, p<.01$.

For different orientations of the head, the same GIFs result in different apparent elevations of the target. Part of the difference may be attributed to altered activity of the otolith organs, and the other part may be attributed to altered activity of proprioceptors in the neck. If conditions are restricted so that the apparent elevation 


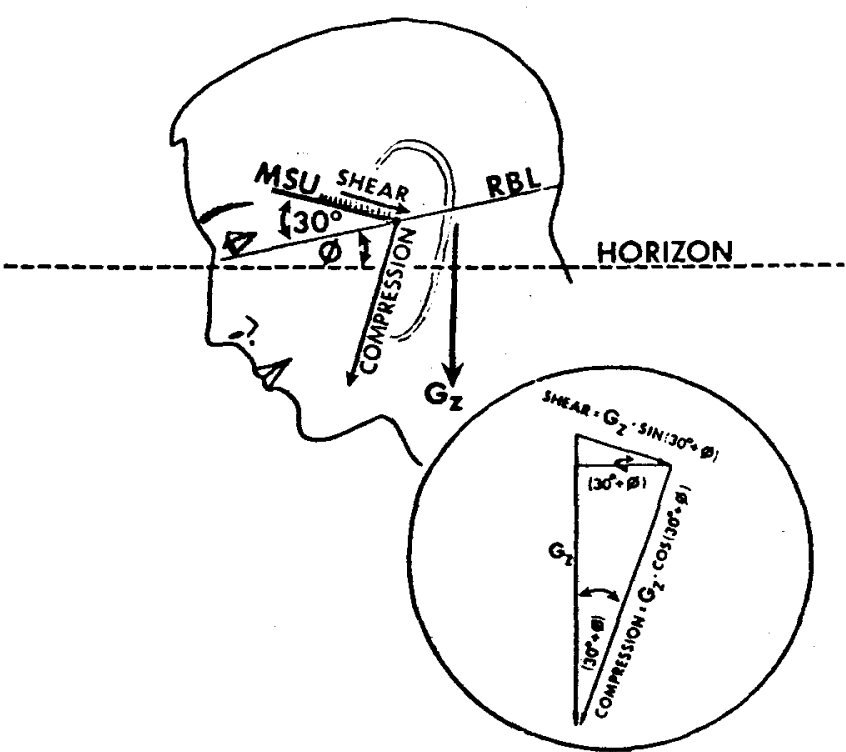

Fig. 4. Anatomical considerations. MSU-macular surface of the utricle; RBL-Reid's baseline; COMPRESSION and SHEAR refer to forces acting at the macular surface of the utricle. The circular insert illustrates quantitative relationships among SHEAR, COMPRESSION, $\mathrm{G}_{2}$, and $\phi$.

of the target depends exclusively on neck proprioception and otolith organ activity, and if neck proprioception is maintained constant while stimulation of the otolith organs is altered, then any changes in the apparent elevation of the target can be attributed to activity of the otolith organs without the confounding influence of neck proprioception. Likewise, if the activity of the otolith organs is maintained constant while neck proprioception is altered, then any changes in the apparent elevation of the target can be attributed to neck proprioception without the confounding influence of otolith organ activity.

The following discussion will employ certain conventions, illustrated in Fig. 4. The symbol, $\phi$, will designate the angle in degrees between Reid's baseline and the horizon. By convention, $\phi$ is negative when the head is pitched forward and positive when the head is pitched backward. The angle at which the macular surface of the utricle is oriented with respect to the horizon is denoted by the quantity $(30 \mathrm{deg}+\phi)$, as measured by Corvera et al (1958). When the head is pitched $30 \mathrm{deg}$ forward, $\phi$ equals $-30 \mathrm{deg}$, and the angle, $(30 \mathrm{deg}+\phi)$, is $0 \mathrm{deg}$.

Schone (1964) has proposed that the apparent elevation of a visual target increases proportionally with shearing forces directed backward along the macular surface of the utricle. Since shearing forces are given by $G_{z}$ sin $(30 \mathrm{deg}+\phi)$, they are eliminated when $\sin (30 \mathrm{deg}+\phi)$ equals 0 , i.e., when the macular surface of the utricle is parallel to the horizon and perpendicular to the GIFs. Also, for any given orientation of the head, the shearing forces should vary linearly with $G_{z}$ at a fixed rate, since $\sin (30 \mathrm{deg}+\phi)$ is a constant for any given value of $\phi$.

If changes in the apparent elevation of the target are proportional exclusively to shearing forces at the macular surface of the utricle, the apparent elevation of the target should define a single line of constant slope when plotted against the shearing forces, i.e., $\mathrm{G}_{\mathrm{Z}} \sin (30 \mathrm{deg}+\phi)$. As seen in the top panel of Fig. 5, a single straight line does not result. Although the slopes computed for the head erect and 15-deg forward pitch conditions do not differ significantly, $t(8)=.47$, the data are discontinuous for the three orientations of the head and result in significantly different $y$ intercepts, $F(2,16)=9.65, p<.01$. Thus, Schone's model does not account adequately for these data; the data indicate differences in apparent elevation that depend on the orientation of the head, but that are independent of shearing forces at the macular surface of the utricle. Clearly, additional variables are implicated.

Correia et al (1968) have reported that shearing forces at the macular surface of the utricle are insufficient to determine the apparent elevation of a visual target. They indicate that compression at the macular surface of the utricle must also be considered, and suggest that a tangent relationship best describes the effects of altered

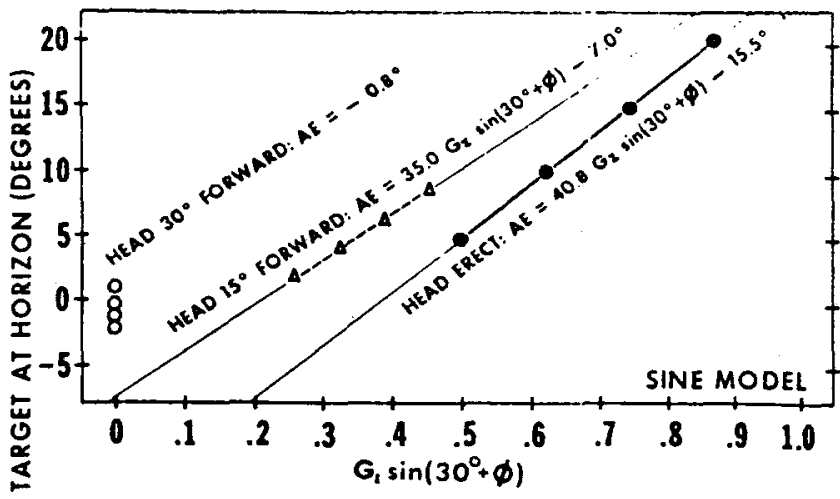

o

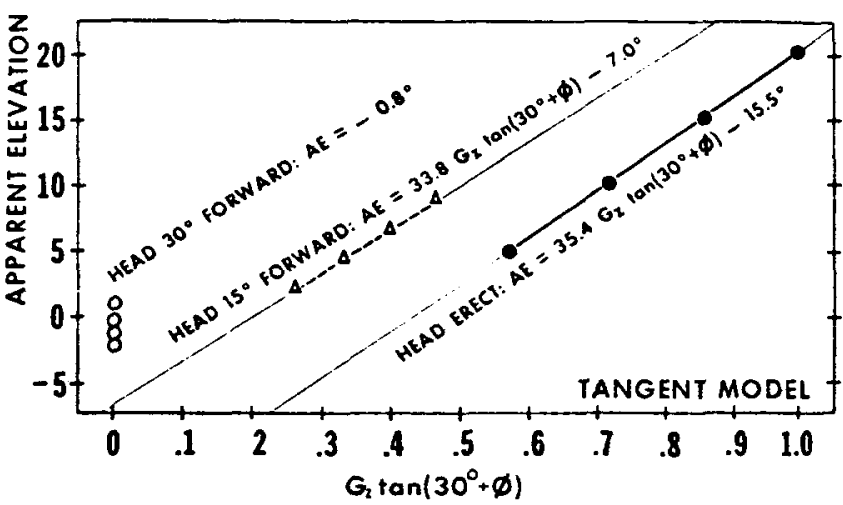

Fig. 5. Apparent elevation of a target at the horizon as a function of head orientation and assumed models of otolith organ activity. AE-apparent elevation of a target at the horizon. The equations for each condition represent the best fit by a linear method of least squares. 
Table 2

Least-Squares Equations for Apparent Elevation

\begin{tabular}{lll}
\multicolumn{1}{c}{ Head Orientation } & \multicolumn{1}{c}{ Sine Model } & \multicolumn{1}{c}{ Tangent Model } \\
Head Erect & $\mathrm{AE}=40.8 \mathrm{G}_{\mathrm{Z}} \sin \left(30^{\circ}+\phi\right)-.5 \phi-15.1^{\circ}$ & $\mathrm{AE}=35.4 \mathrm{G}_{\mathrm{Z}} \tan \left(30^{\circ}+\phi\right)-.5 \phi-15.1^{\circ}$ \\
& $\mathrm{AE}=20.4\left(\mathrm{G}_{\mathrm{Z}}\right) \mathrm{G}_{\mathrm{Z}}-15.1^{\circ}$ & $\mathrm{AE}=20.4\left(\mathrm{G}_{\mathrm{Z}}\right) \mathrm{G}_{\mathrm{Z}}-15.1^{\circ}$ \\
Head Pitched $15^{\circ}$ Forward & $\mathrm{AE}=35.0 \mathrm{G}_{\mathrm{Z}} \sin \left(30^{\circ}+\phi\right)-.5 \phi-15.1^{\circ}$ & $\mathrm{AE}=33.8 \mathrm{G}_{\mathrm{Z}} \tan \left(30^{\circ}+\phi\right)-.5 \phi-15.1^{\circ}$ \\
& $\mathrm{AE}=9.1\left(\mathrm{G}_{\mathrm{Z}}\right) \mathrm{G}_{\mathrm{Z}}-7.6^{\circ}$ & $\mathrm{AE}=9.1\left(\mathrm{G}_{\mathrm{Z}}\right) \mathrm{G}_{\mathrm{Z}}-7.6^{\circ}$ \\
& $\mathrm{AE}=-.5 \phi-15.1^{\circ}$ & $\mathrm{AE}=-.5 \phi-15.1^{\circ}$ \\
Head Pitched $30^{\circ}$ Forward & $\mathrm{AE}=-0.1^{\circ}$ & $\mathrm{AE}=-0.1^{\circ}$ \\
\hline
\end{tabular}

GIFs on the apparent elevation of a visual target.

If changes in the apparent elevation of the target were proportional exclusively to $G_{z} \times$ the ratio of shear to compression, i.e., $G_{z} \times$ the quantity, $\left[\left(G_{z}\right.\right.$ sin $\left.(30 \mathrm{deg}+\phi)) /\left(G_{z} \cos (30 \mathrm{deg}+\phi)\right)\right]$, which equals $G_{z} \times$ $\tan (30 \mathrm{deg}+\phi)$, the apparent elevation of the target should define a single line of constant slope when plotted against $G_{z} \tan (30 \mathrm{deg}+\phi)$. As seen in the bottom panel of Fig. 5, a single straight line does not result. The data show differences in apparent elevation that depend on the orientation of the head but that are independent of the ratio of shear to compression. Again, additional variables are implicated.

Both the sine and the tangent models result in a different $y$ intercept for each orientation of the head. According to the assumptions of both models, the $y$ intercept represents where a visual target at the horizon would appear to be located when otolith organ activity is eliminated. For both models, the $y$ intercept is $-15.5 \mathrm{deg}$ when the head is erect, $-7 \mathrm{deg}$ when the head is pitched $15 \mathrm{deg}$ forward, and $-0.8 \mathrm{deg}$ when the head is pitched $30 \mathrm{deg}$ forward. Since the $\mathrm{y}$ intercept is different for different orientations of the head, both models should include a term to account for the role of neck proprioception.

A method of least squares linear fit to the data shows that the $y$ intercept can be expressed as $-.5 \phi-$ $15.1 \mathrm{deg}$. By substituting the expression, $-.5 \phi-$ $15.1 \mathrm{deg}$, for the $\mathrm{y}$ intercepts of the previously derived equations of Fig. 5, we generate the new equations presented in Table 2.

Under the assumption that the mean computed slope is the best estimate of the change in apparent elevation as a function of otolith organ activity, the equations of Table 2 reduce to

$$
\mathrm{AE}=37.9 \mathrm{G}_{\mathrm{z}} \sin (30 \mathrm{deg}+\phi)-.5 \phi-15.1 \mathrm{deg}
$$

and

$$
\mathrm{AE}=34.6 \mathrm{G}_{\mathrm{z}} \tan (30 \mathrm{deg}+\phi)-.5 \phi-15.1 \mathrm{deg}
$$

for the sine and tangent models, respectively.

As illustrated in Fig. 6, these new models, incorporating separate terms for the effects of otolith organ activity and neck proprioception, accommodate the data quite well, accounting for $96.3 \%$ and $98.4 \%$ of the variance of the means with the sine and tangent models, respectively.

\section{CONCLUSION}

This experiment has indicated that the apparent elevation of a visual target can be expressed as the additive combination of two processes, one dependent on otolith organ activity, (Ot), and the other, on neck proprioception, (Np).

$$
\mathrm{AE}=(\mathrm{Ot})+(\mathrm{Np})
$$

The activity of the otolith organs was viewed as a function of both the intensity of the GIFs, given by $G_{z}$, and the anatomical orientation of the macular surface of
Fig. 6. Measured vs predicted apparent elevation of a target at the horizon for two models of the combined effects of otolith organ activity and neck proprioception.
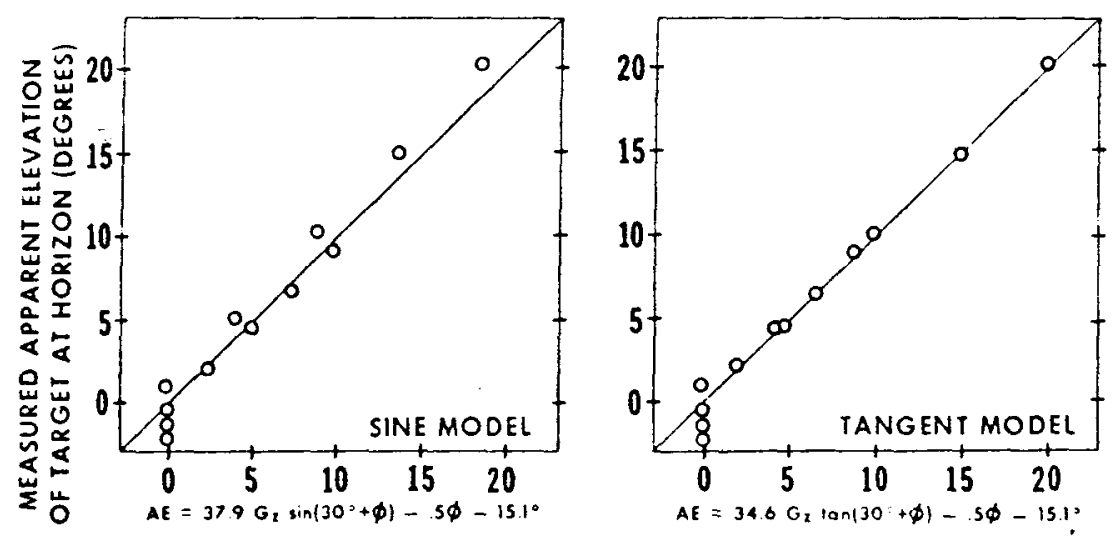

PREDICIED APPARENT ELEVATION OF TARGET AT HORIZON (DEGREES) 
the utricle relative to the GIFs, given by the quantity, $(30 \mathrm{deg}+\phi)$.

$$
(\mathrm{Ot})=\mathrm{F}\left(\mathrm{G}_{\mathrm{z}}, \phi\right)
$$

Neck proprioception was considered to be a function of the angle at which the head is pitched relative to the trunk: this angle is equivalent to $\phi$ when GIFs are applied along the $G_{z}$ body axis.

$$
(\mathrm{Np})=\mathrm{J}(\phi)
$$

Two altemate, explicit, and quantitative models concerning the influences of otolith organ activity and neck proprioception in the elevator illusion were developed; both models provided a reasonably close approximation to the data. Additional research, employing a wider range of $\phi$ and $G_{z}$, will be needed to evaluate the alternative models proposed here.

\section{REFERENCES}

Beckh, H. von. Gravity changes in aircraft and ships. Journal of the British Interplanetary Society, 1956, 15, 73-81.

Brandt, U., \& Fluur, E. Postural perceptions and eye displacements produced by a resultant vector acting in the median sagittal plane of the head. I: Responses along three axes by stepwise increasing $\phi$ with the subject heading centripetally in an erect and tilted position. Acta Oto-laryngologica, 1967a, 63, 489-502.

Brandt, U., \& Fluur, E. Postural perceptions and eye displacements produced by a resultant vector acting in the median sagittal plane of the head. II: Continuous responses along the $\mathrm{Y}$ axis with the subject in a vertical position heading centripetally and centrifugally. Acta Oto-laryngologica, $1967 \mathrm{~b}, 63,564-578$.

Christy, R. L. The new human centrifuge. Journal of Aviation Medicine, 1949, 20, 454-458.

Clark, B., \& Graybiel, A. Influence of contact cues on the perception of the oculogravic illusion. Acta Oto-laryngologica, $1968,65,373-380$.

Clark, B., Graybiel, A., \& MacCorquodale, K. The illusory perception of movement caused by angular acceleration and by centrifugal force during flight. II. Visually perceived motion and displacement of a fixed target during turns. Journal of Experimental Psychology, 1948, 38, 298-309.

Cohen, L. A. Role of eye and neck proprioceptive mechanisms in body orientation and motor coordination. Journal of Neurophysiology, 1961, 24, 1-11.

Cohen, M. M. Sensory-motor adaptation and after-effects of exposure to increased gravitational forces. Aerospace Medicine, 1970a, 41, 318-322.
Cohen, M. M. Hand-ey'e coordination in altered gravitational fields. Aerospace Medicine, 1970b. 41, 647-649.

Cope, S., \& Ryan, G. M. S. Cervical and otolith vertigo. Journal of Laryngology, 1959, 73, 113-120.

Correia, M. J., Hixson, W. C., \& Niven, J. I. On predictive equations for subjective judgments of vertical and horizon in a force field. Acta Oto-laryngologica, 1968, Monograph Supplement 230.

Corvera, J., Hallpike, C. S., \& Schuster, E. H. J. A new method for the anatomical reconstruction of the human macular planes. Acta Oto-laryngologica, 1958, 49, 4-16.

Crosbie, R. J. Directional control of accelerative forces in a centrifuge by system of gimbals. Journal of Aviation Medicine, 1956. 27, 505-511.

Crosbie, R. J. General description and performance characteristics of the human centrifuge. NADC Report NADC-MR-6721, Naval Air Development Center, Warminster, Pennsylvania, 1967.

DeLage, Y. Études expérimentales sur les illusions statiques et dy namiques de direction pour servir à déterminer les fonctions des canaux demicirculaires de l'oreille interne. Archives de Zoologie Experimentale et Generale, 1886, 4, 535-624.

Fluur, $E$. The interaction between the utricle and the saccule. Acta Oto-laryngologica, 1970, 69, 17-24.

Graybiel, A. Oculogravic illusion. A.M.A. Archives of Ophthalmology, 1952, 48, 605-615.

Graybiel, A., \& Clark, B. Validity of the oculogravic illusion as a specific indicator of otolith function. Aerospace Medicine, $1965,36,1173-1181$.

Graybiel, A., Clark, B., \& MacCorquodale, K. The illusory perception of movement caused by angular acceleration and by centrifugal force during flight. I. Methodology and preliminary results. Journal of Experimental Psychology. $1947,37,170-177$.

Guedry, F. E. Some vestibular problems related to orientation in space. Acta Oto-laryngologica, 1968, 65, 174-185.

Kleyn, A. de. Action réflexes du labyrinthe et du cou sur les muscles de l'oeil. Archives Neerlandaises de Physiologie de l'homme et des animaux, 1918, 2, 644-649.

Niven, J. I.. Whiteside, T. C. D., \& Graybiel, A. The elevator illusion: Apparent motion of a visual target during vertical acceleration. Bureau of Medicine \& Surgery Report No. 89, U.S. Naval Aviation Medical Center, Pensacola, Florida, 1963.

Roman, J. A., Warren, B. H., Niven, J. I., \& Graybiel, A. Some observations on the behavior of a visual target and a visual afterimage during parabolic flight maneuvers. Aerospace Medicine, 1963, 34, 841-845.

Schone, $H$. On the role of gravity in human spatial orientation. Aerospace Medicine, 1964, 35, 764-772.

Whiteside, T. C. D. Hand-eye coordination in weightlessness. Aerospace Medicine, 1961, 32, 719-725.

(Received for publication February 5, 1973; accepted March 30, 1973.) 